\title{
Dynamical real numbers and living systems
}

\author{
Dhurjati Prasad Datta* \\ Department of Mathematics, North Bengal University, \\ P.O. North Bengal University, Darjeeling, India, Pin: 734430
}

\begin{abstract}
Recently uncovered second derivative discontinuous solutions of the simplest linear ordinary differential equation define not only an nonstandard extension of the framework of the ordinary calculus, but also provide a dynamical representation of the ordinary real number system. Every real number can be visualized as a living cell -like structure, endowed with a definite evolutionary arrow. We discuss the relevance of this extended calculus in the study of living systems. We also present an intelligent version of the Newton's first law of motion.
\end{abstract}

Chaos, Solitons, and Fractals, vol 20, issue 4, 705-712, (2004)

*email:dp_datta@yahoo.com 


\section{Introduction}

Let $t$ be a real variable. Then a key assumption in the formalism of mathematical analysis (calculus), and hence in classical and quantum dynamics, is that $t$ changes only by (linear) translations. The variable $t$ assume values from the set of real numbers, imagined as fixed (static), ideal mathematical objects. The fundamental concept of limit, as treated in the standard $\epsilon, \delta$-definition, disregards the inherent dynamical aspect of the concept, by reducing it to a relative statement between 'sufficiently small' real numbers. The growth of the modern (standard) analysis, thus not only hides the deep interrelationship between analysis and dynamics, but also rejects infinitesimally small (infinitely large) numbers. The nonstandard analysis of Robinson [1] , although puts the theory of infinitesimally small numbers into a sound mathematical basis with interesting applications in the theory of fractals and stochastic analysis [2], the true dynamic features of the infinitesimals have not been appreciated. The recently discovered second derivative discontinuous scale free solutions of the linear ordinary differential equations (of ordinary calculus) [3, 4] now provides, in one hand, a new direct proof of the existence of infinitely small numbers in the set of real numbers, and on the other, shows that such numbers must be 'intrinsically dynamic' in the sense that they are in a state of spontaneous random fluctuations, which, in turn, induces a sense of evolution to every ordinary real number. The qualifier 'intrinsic' means that the said evolution is not (actually it can not be) due to any externally applied force, as it is necessary in the treatment of ordinary dynamics, but instead an intrinsic property of the real number system itself. In this paper, we point out a few more interesting features of the dynamical number system and argue that the extended 'calculus of dynamical numbers' should be the ideal framework for the study of living (intelligent) systems. We also give an intelligent version of the Newton's first law of motion, which should be considered as the 'first law of motion' for living systems. Let us recall here that the formalism of calculus (and dynamics) was originally invented to study the motion of material bodies, the planetary system, for instance. The modern studies of living systems (life sciences) tend in fact to reduce 'life' essentially to biochemical (physical) processes, although there have been occasional suggestions for the need of an intelligent principle, rather than simply material (physical) principles as emerged from the studies of quantum field theories and nonlinear sciences, to understand the complex dynamics of a living system [5]. The main theme of the present paper is therefore to highlight the potentially interesting dynamical features of the new scale free calculus in the context of a living intelligent system.

The singular role of fractals in the studies of nonlinear systems is now well appreciated. Recently, there have been a lot of studies devoted to applications of fractal geometry, deterministic chaos and stochastic dynamics in classical and quantum dynamics [6, 2, 7, 8, 9], as 
well as in biological systems [10]. However, the inherent dynamic nature of any fractal object, though implicitly present in their definitions ( as the limit set of an infinite iterative process, inducing a temporal sense), is yet to be enunciated clearly. Our results indicate that any dynamical system, being inherently scale free and directed, must tend toward a random fractal like evolutionary process, with approximate (statistical) self-similarity over multiple random scales. Conversely, the emergence of fractal objects must be viewed in an evolutionary setting, provided naturally by the scale free extension of calculus. Our work also tells that points in the real number line, and hence in the space-time manifold of any physical theory, have a Cantorian structure, but this need not necessarily be linked only with the Planck scale physics, as in the $\mathcal{E}^{\infty}$ theory [7], but may arise more universally and naturally to any truly dynamical theory, for instance, in the context of living systems. The possibility of random fractal topology, as an inherent manifestation of stochastic dynamics near any fixed point of the underlying quantum field theory, has recently been pointed out «11, 13, 14].

\section{Scale free calculus}

\subsection{Generalized Solutions}

Let us begin by reviewing the important results of the new scale free calculus [3, 4]. In ordinary (linear) calculus, one begins with an independent real variable $t$ (say) and then introduces the concepts of limit, continuity, derivatives and so on for a function of $t$. For definiteness, the variable $t$ is said to define the $\mathrm{O}(1)$ scale of ordinary (macroscopic/ coarse grained) real numbers. The scale free extension of calculus, however, is defined on the basis of scale free, second derivative discontinuous solutions of the linear differential equation

$$
\frac{\mathrm{d} T}{\mathrm{~d} t}=T
$$

which is given by

$$
\ln T(t)=t+r k \phi\left(t_{1}\right), \phi=t_{1} \tau\left(t_{1}^{-1}\right)
$$

where $\tau\left(t_{1}\right)=T\left(\ln t_{1}\right), t_{1}=k_{0} t, k_{0}>0$ being a solution of eq (1), but in the log scale, denotes a self -similar replica of $T(t)$ in the smaller logarithmic variable $\ln t_{1}^{-1}$ and $k$ and $k_{0}$ are arbitrary scaling parameters. Here $r$ is a random variable assuming values from $\{+1,-1\}$ with equal probability, say. The solution is scale free, since both $\tau$ and $\phi$ are solutions of scale free equations

$$
t \frac{\mathrm{d} \tau}{\mathrm{d} t}=\tau
$$

and

$$
t \frac{\mathrm{d} \phi}{\mathrm{d} t}=0
$$


respectively. Clearly, $T(t)$ satisfies eq(11) if and only if $\phi$ satisfies eq(4), which means, in turn, that $\tau$ must satisfy eq(3). The scaling parameter $k_{0}$ is representative of this scale free nature. Utilizing the scaling law $\tau(k t)=k \tau(t)$, and the scale free property, one can always re-scale $\tau$ suitably to make $k$ arbitrarily small. Further, the generalized solution is singular at $t=0$ and intrinsically nonlocal since the implicit definition of the generalized function $T$ involves distinct points $t$ and $\ln t_{1}^{-1}$, for instance, over two different scales. These two properties viz., the singularity and nonlocality, tell that $T(t)$ is a new, nontrivial solution of eq(1)). (The well known standard solution of eq (1) $\ln T_{s}(t)=t+c, c$ being an ordinary constant, is local (i.e., defined at a single point) and well defined for all $t$ in the real number set $R$. Moreover, replacing $\tau$ in eq(2) by the standard solution $\tau=c t$ (say), one reproduces the standard solution $T_{s}$, but in $R-\{0\}$. Consequently, the standard solution belongs to this more general class of solutions, when the initial condition at $t=0$ is defined only approximately. In other words, the standard solution is only an approximate solution, in the context of this more general class of solutions. Further, the framework of ordinary calculus does not allow randomness in the context of eq(1).) The nontrivial scale free solution $\phi$ of eq (4) is called a slowly varying generalized constant, since $\phi$ remains constant for any finite $t$, but may experience a slow variation on the infinitesimal scale $\ln t_{1}^{-1}$, for an arbitrarily large $t$.

The nontrivial part of the generalized solution $\ln T$ corresponds to infinitesimally small random scales. In fact, even as the continuous (ordinary) real variable $t$ exhausts all arbitrarily small ordinary real numbers in the vicinity of $t=0, \ln T(0)=\lim _{t \rightarrow 0} k \phi(t)$ remains arbitrarily small but nonzero, for an arbitrarily small but nonzero generalized constant $k$. Note that $k$ can not vary faster than $\phi$, by definition. Further, it is useful to model the arbitrariness of the scale factor $k$ by a continuous random variable $K$ which can assume values $k$ with a uniform probability density function, for instance. However, as shown in Ref[3], a Gammalike distribution seems to be an appropriate distribution in the context of the above scale free solutions.

We note, further, that the singularity of $\phi$ is not specific to $t=0$ only. In fact, by the translation symmetry of eq(1) $, \phi\left(t^{\prime}\right)=\phi(t), t^{\prime}=t-t_{0}$, so that $(\mathrm{i}) \phi(t)\left(=1+k \phi\left(t_{1}\right)\right)$ is actually singular at every point $t_{0}$ in the ordinary real axis and (ii) is essentially a universal random function in the neighbourhood of 1 ( $k \phi$ is infinitesimal in the neighbourhood of 0 ), where both $t$ and $t_{1}\left(t \neq t_{1}\right)$ are random variables defined near 1 . Thus, $\lim _{t \rightarrow t_{0}}\left(\ln T(t)-t_{0}\right)=\ln T(0)(\neq 0)$. In the ordinary calculus, this limit must necessarily equal to 0.

Definition 1: As the limit $t \rightarrow 0$ exhausts all the coarse grained real numbers, there exists a continuum of random, infinitesimally small microscopic positive numbers (scales) given by $\eta=k \phi\left(t_{1}\right), k>0$. Clearly, $\eta \neq 0$ is less than any arbitrarily small, nonzero, coarse grained, 
positive real number.

Remark 1: The standard solution of eq (11) $\ln T_{s}(t)=t, T(1)=e$ defines a 1-1 (identity) mapping of the real number set $R$ onto itself. The generalized solution $\ln T(t)$ now defines an extension of the identity mapping from $R$ to the nonstandard real set $\mathbf{R}$. Clearly, $\mathbf{R}=R$, $T$ being an exact solution of eq (1). Accordingly, every ordinary real number $t$ is identified (extended) with (to) the set (actually an equivalence class under the relation 'infinitely close neighbour') of scale free (fat) real numbers denoted $\mathbf{t}=t+r N_{0}$, where $N_{0}$ is the nontrivial neighbourhood of positive infinitesimals $N_{0}=\{\eta\}$. The macroscopic zero 0 , for instance, corresponds to the set of scale free (first order) infinitesimals $\mathbf{0}=\{r \eta\}(<\mathbf{0}>=0)$. Here, $<>$ denotes the expectation value of the random variable t. Determining the exact value of a real number is thus theoretically impossible, because of the identity $\mathbf{R}=R$. Further, the additive zero of $\mathbf{R}$ now corresponds to the set of higher order infinitesimals, viz., $0=\left\{\eta^{2}, \eta^{3}, \ldots\right\}$.

Remark 2: The coarse grained variable $t$ undergoes changes by linear translations. The intrinsic randomness of the infinitesimals now tells that the infinitely small numbers are in a state of continual fluctuations, analogous to a system of ideal gas in thermodynamic equilibrium, say. The fluctuations in the neighbourhood of the normalized class $\mathbf{1} \equiv \mathbf{t} / t$ could be modeled by the inversion rule $t_{-} \rightarrow t_{-}^{-\alpha}=t_{+}^{\alpha}$ where $\alpha>0$ and $t_{ \pm}=1 \pm \eta, \eta$ being an (first order) infinitesimal so that $\mathrm{O}\left(\eta^{2}\right)=0$, by definition. The points in the infinitesimal neighbourhood of 1 can therefore change by discrete jumps (inversions), the length of jumps being random because of the arbitrariness of $\alpha$. That inversion transformations should be operative here is actually traced to the non-locality of the generalized solutions (for a more direct proof see below). These small scale fluctuations are, however, un-noticeable at the level of the macroscopic scale, so that $t=<\mathbf{t}>$ behaves as a smooth linear variable. We also note that $<\phi(t)>=<\phi\left(t^{-1}\right)>=<$ $1>=1$.

To prove the second derivative discontinuity of the generalized solution and to establish its directed and irreversible character, let us now present a simpler solution of eq(3) displaying the possibility of inversion in an explicit manner.

\subsection{Second derivative discontinuity and Irreversibility}

Let $t_{ \pm}=1 \pm \eta, 0<\eta<<1$, so that $\mathrm{O}\left(\eta^{2}\right)=0$ and $t_{-} \rightarrow t_{-}^{-1}=t_{+}$. Let also that $\tau\left(t_{ \pm}\right)=\tau_{ \pm}$. Then the function $\tau_{f}(t)$ defined by

$$
\tau_{f}(t)=\left\{\begin{array}{ll}
\tau_{-} & \text {if } t \lesssim 1 \\
\tau_{+} & \text {if } t \geqq 1
\end{array}, \tau_{-}\left(t_{-}\right)=1 / t_{+}, \tau_{+}\left(t_{+}\right)=t_{+}\right.
$$

constitutes a new nontrivial solution of eq(3) , in the neighbourhood of $t=1$, with the initial condition $\tau_{f}(1)=1$. Indeed, it is easy to verify, using $\mathrm{d} t_{-}=-\mathrm{d} t_{+}$(and/or $t_{-}^{-1} \mathrm{~d} t_{-}=-t_{+}^{-1} \mathrm{~d} t_{+}$, since $\left.\ln t_{-}=-\ln t_{+}\right)$, that 


$$
\frac{\mathrm{d} \tau_{-}}{\mathrm{d} t_{-}}=-\frac{\mathrm{d} \tau_{-}}{\mathrm{d} t_{+}}=1 / t_{+}^{2}, \frac{\mathrm{d} \tau_{+}}{\mathrm{d} t_{+}}=1
$$

but

$$
\frac{\mathrm{d}^{2} \tau_{-}}{\mathrm{d} t_{-}^{2}}=2 / t_{+}^{3}, \frac{\mathrm{d}^{2} \tau_{+}}{\mathrm{d} t_{+}^{2}}=0
$$

which tell that the function $\tau_{f}(t)$ defined by eq(5) has continuous first derivative at and in the vicinity of $t=1$, but its second derivative has a discontinuity at $t=1$. One notes, moreover, that eq([6]) can be written as

$$
t_{-} \frac{\mathrm{d} \tau_{-}}{\mathrm{d} t_{-}}=\tau_{-}, t_{+} \frac{\mathrm{d} \tau_{+}}{\mathrm{d} t_{+}}=\tau_{+}
$$

so that $\tau_{f}(t)$ is a solution. Because of its second derivative discontinuity, it is indeed a new solution, distinct from the infinitely differentiable standard solution.

A fundamental property of the new solution eq(5), which is exact in the extended $\mathbf{R}$ with nontrivial infinitesimals, is that it breaks the reflection (time reversal/parity) symmetry of the underlying equation (3). (The solution is approximate $\left(\mathrm{O}\left(\eta^{2}\right)\right)$ in the ordinary real number set $R$. The origin of the second derivative discontinuity is then traced to the approximation introduced by the substitution $t_{-}^{-1}=t_{+}$. In $\mathbf{R}$, however, this approximation is an intrinsic property of the infinitely small numbers, which can not be improved to an unlimited accuracy. In the Appendix, we present an exact (to all orders of $\eta$ ) random solution of eq(3).) Let $P: P t_{ \pm}=t_{\mp}$ denote the parity transformation close to $t=1$. Then the parity reversed function $\tau_{f}^{P}: \tau_{-}^{P}=t_{-}, \tau_{+}^{P}=1 / t_{-}$is also a solution of eq(3), but, clearly it is different from the original solution $\tau_{f}$. We note, in particular, that $\tau_{f}^{P}$ diverges as $\eta \rightarrow 1^{-}$, in contrast to $\tau_{f}$ which is well-behaved, as it should be. Consequently, one expects that the solution $\tau_{f}$ is more likely to materialize, compared to the parity reversed solution. As a result, it is more natural for the variable $t$ to change from $t_{-}$to $t_{+}$(by an instantaneous jump) via $t_{-} \rightarrow t_{-}^{-1}=t_{+}$, since the solution (5) admits the transition $\tau_{-} \rightarrow \tau_{+}=1 / \tau_{-}$, and then is forced to increase with $\eta \rightarrow 1^{-}$until a second generation inversion in the small scale variable $\eta$, rather than to change alternatively. Consequently, the solution eq(5) tells in a succinct way that the function $\tau_{f}(t)$ must always be monotonically increasing and hence is directed irreversibly in the forward direction.

We note that the problem of time asymmetry (arrow of time) in Physics is that all the fundamental equations of motion are time reversal symmetric. Consequently, one expects that the corresponding solutions must also respect the symmetry of the underlying equation and so must be time reversal symmetric. The present solution is the first explicit example which fails to respect the symmetry of the underlying equation, analogous to the spontaneous (vacuum) symmetry breaking encountered in the quantum field theory. As advocated many a times by 
Prigogine [12] (and also implicit in El Naschie's [8] work), any time reflection symmetric system must essentially be interpreted as devoid of an intrinsic time sense. In this sense a state in thermal equilibrium is timeless since it lacks an intrinsic tendency of evolution toward a final state. Accordingly, a true time variable should be one which breaks this reflection symmetry spontaneously. Our solution naturally stands out as a genuine candidate for time.

Remark 3: The solution (5) shows explicity that inversion $t_{\mp}=1 / t_{ \pm}$is as natural a mode of 'increment' (change) for a real variable, as it is usually assigned to translation. Recall that the standard solution of eq(3) under pure translation (i.e., when $t$ is allowed to change only by translation) is

$$
\tau\left(t_{-}\right)=t_{-}, \tau\left(t_{+}\right)=t_{+}
$$

We note here also that another exact solution of eq(3) (under inversion) is

$$
\tau_{-}=1 / t_{+}, \tau_{+}=1 / t_{-}
$$

Comparing the above equation with eq(河), we see that in eq(河) only 'one sided inversion' is realized, whereas eq(10) accommodates inversions in both the ways. Moreover, solution (10) is an effect of pure inversion only, when eq(5) is generated by accommodating both the translation and inversion modes of change for $t$. One also verifies that eq(10) is infinitely differentiable, as in eq(9), and is time reflection symmetric. The solution (10) thus has the natural status of 'equlibrium' infinitesimal fluctuations near $t=1$. We recall that the equilibrium fluctuations in thermodynamics is time symmetric (c.f., remark 2).

Remark 4: A more general class of time asymmetric solution of eq(3)

$$
\tau_{f}^{\alpha}(t)=\left\{\begin{array}{ll}
\tau_{-} & \text {if } t \lesssim 1 \\
\tau_{+} & \text {if } t \geqq 1
\end{array}, \tau_{-}\left(t_{-}\right)=t_{+}^{-\alpha}, \tau_{+}\left(t_{+}\right)=t_{+}, \alpha>0\right.
$$

when $t_{-}$relates to $t_{+}^{\prime \alpha}=1+\alpha \eta^{\prime}>1$ via $t_{-} \rightarrow t_{-}^{-\prime \alpha}=t_{+}^{\prime \alpha}$ so that $\mathrm{d} \eta=\mathrm{d} \eta^{\prime} 1$ Clearly, the solution (11) reduces to eq(可) for $\alpha=1$. We note that a power law reduces to a scaling (dilaton) transformation for small enough $\eta: \eta_{1}=\alpha \eta$ near $t=1$, satisfying $0<\alpha \eta<<1$. One can also verify that the solution (11) is only first order differentiable, the second derivative having a discontinuity at $t=1$. It also follows that a real variable $t$ can not only change, in the vicinity of $t=1$, by a pure inversion $\alpha=1$, but also undergo changes by discrete random jumps, length of a jump being determined by the arbitrary parameter $\alpha$. Thus a real variable $t(\lesssim 1)$, (viz. $\left.t_{-}=1-\eta\right)$, for instance, can now connect instantaneously to a spectrum of values $t_{\alpha+}=1+\alpha \eta$, which can naturally be assumed to be distributed following a probability law. In other words,

\footnotetext{
1 The case $\alpha<0$ is also interesting. Two points $t_{1}=1+\eta_{1}$ and $t_{2}=1+\eta$, on the same side of 1 , may be related by an infinitesimal scaling $t_{1}=t_{2}^{\beta}, \eta_{1}=\beta \eta, \alpha=-\beta<0$. The corresponding solution is $\tau\left(t_{1}\right)=t_{2}^{\beta}, \tau\left(t_{2}\right)=t_{2}$.
} 
the existence of this class of solutions puts our original assumption of fluctuating infinitesimals in the neighbourhood of a real number [3] in a farmer footing.

The above class of time asymmetric solutions now motivates the

Definition 2: Let $T(t)=t \phi\left(t_{1}\right)=t\left(1+k t_{1}^{-1} \tau_{f}\left(t_{1}\right)\right)$. The variable $T$ is intrinsically time-like if and only if $\tau_{f}$ belongs to the class of solutions given by eq(15) (equivalently, eq(11).

Remark 5: In case $\tau_{f}$ belongs either to the solution (9) or (10) the corresponding $T$ would be reflection symmetric (as well as infinitely differentiable) and can not be considered as a genuine time variable. We note that the contemporary dynamical theories make use of a time variable belonging to the solution (9) only. We note in this context that the conventional notations $t$ and $x$ for time and space variables can not distinguish the two types of variables in an intrinsic way, since both the variables are reflection symmetric solution (9) of eq(3).

As a consequence of the above remarks, one can restate the definition 1 thus

Definition 3: A variable $t$ is macroscopic if it belongs to the trivial reflection symmetric solution (9). A variable $\eta$ defined by $\left.\eta=k t_{1}^{-1} \tau_{f}\left(t_{1}\right)\right), t_{1}=t / k, t \rightarrow 0,0<k<<t$ being an arbitrarily small random variable, so that $\mathrm{O}\left(\eta^{2}\right)=0$, is a small scale microscopic variable when $\tau_{f}$ stands for the reflection symmetric fluctuating solution (10).

To summarize, we have presented two classes of nontrivial, exact solutions (5) and (10), besides the standard solution (9), of the scale free equation (3), in the extended $\mathbf{R}$. The solution (10) is infinitely differentiable and reflection symmetric, as is eq(9), but eq(5) is second derivative discontinuous and breaks the reflection symmetry spontaneously. Accordingly, the generalized solution (2) of eq(11) is either reflection symmetric or otherwise, depending on the nature of the scale free component involving $\tau$ in its definition.

Consequently, every real number is associated with a nontrivial neighbourhood of infinitesimally close elements $\mathbf{t}=t(1+\eta)$, which would have been in a state of 'equilibrium' random fluctuations, according to the definition 3, had there been only reversible, infinitely differentiable solutions (91) and (10). Because of the second derivative discontinuous, asymmetric solution (5), the equilibrium, scale free fluctuation would be generically broken, inducing an irreversible, spontaneous evolution, albeit infinitely slow, to any approximate evaluation of the real number $t$. Note that any element in the set $\mathbf{t}$ is an approximation of $t$, for a nonzero $\eta$. As shown in detail in Ref[3, 4], this slow, intrinsic evolution would ultimately lead $\eta$ to approach the golden mean number $\frac{\sqrt{5}-1}{2}$, following the approximants of the corresponding continued fraction. ( The origin of the golden mean could be seen as follows. Being intrinsically an increasing variable (see below), the initially small variable $\eta$ would grow to a $\eta_{-} \lesssim 1$. Following the solution (5), $\eta_{-}$would then be replaced by $\eta_{+}^{-1}=1+\eta_{1}, \eta_{1}$ being another small increasing variable and so on. The intrinsic evolution thus drives $\eta$ to follow the cascades of the golden 
mean continued fraction $1 /(1+1 / 1+\ldots))$. The existence of the second derivative discontinuous solutions, with nontrivial dynamical properties, should be of crucial importance for future studies of dynamics. Our studies may only be considered as a few initial steps towards this direction.

One may offer a 'minimality argument' in support of the inevitability of the time asymmetric solution in the extended solution space of eq(3). Note that in solving a first order (linear) differential equation (with $C^{\infty}$ data), the minimum condition one needs to meet is that the solution should at least be first order continuously differentiable. The infinitely differentiable solution (9) (and also (10)), however, corresponds to a situation where the information (knowledge) regarding the infinite differentiability of the solution is simply wasted. On the other hand, the time asymmetric solution just satisfies the minimal condition of being only the first derivative continuous. Now, invoking the common sense principle, viz., the minimum information wastage (loss) means the maximum opportunity of progress, one may assert that in the 'universe' of all possible solutions of eq(1), the time asymmetric solution would be naturally selected, because the said solution possesses the potentiality of growing into a more complex fractal like structure over a period of 'infinitely' long time. Accordingly, the time asymmetric solution offers a dynamical system the maximum opportunity of evolution and growth. We recall that the time sense is also generated here intrinsically by the solution itself. We note also that because of the intrinsic randomness and approximate nature of $\mathbf{R}(=R)$, even the symmetric (fluctuating) solution (10) fails to return an initial value $t_{-}$(say) exactly, viz., $t_{-} \rightarrow t_{+} \rightarrow t_{-}^{\prime}$, $t_{-}^{\prime}$ being only approximately equal to $t_{-}$. This slight mismatch between the initial and final values would definitely induce a symmetry breaking, thereby realizing the time asymmetric solution in due course. Consequently, the time asymmetric solution is uniquely realized in the extended scale free calculus.

Before closing this section, let us note that the above analysis reveals an intrinsic mechanism of change and evolution, which should universally be present not only in any dynamical system, but even rooted indelibly to the most fundamental of all mathematical entities, viz., the real number system. In other words, the concepts of intrinsic evolution and time sense turn out to be truly fundamental at par with real numbers, sets etc. We note that in the ordinary framework of dynamical theories, motion and changes are meaningful only under the influence of an externally imposed force (or force field). Consequently, the framework of the established dynamical theories such as Newtonian mechanics, Einstein's general relativity, quantum mechanics and so on, needs to be extended suitably incorporating this universally present intrinsic evolution [11, 13, 14]. More fundamentally, the study of the scale free calculus, as initiated in this series of works, may likely to provide a truly unified framework to understand natural and 
biological processes at different length, time and energy scales, in a scale invariant manner.

\section{Dynamical numbers and cell division}

Natural numbers are most primitive of all numbers, being invented by primitive men to keep records of the number of objects they possessed. As such, natural numbers, and for that matter any number, are abstract mathematical concepts, which reside permanently and unchangeably in the 'timeless' world of concepts. At an empirical level, however, a natural number $n$ (say) is associated with the cardinal number of the set of $n$ number of natural objects (we disregard the subtle distinction between the cardinal and ordinal numbers). For example, 2 is associated with the set of 2 billiard balls and so on. Instead of the set of billiard balls, one could have related 2 to the set of 2 flowers as well. Empirically, a set of living objects, e.g., a set of flowers, are prone to undergo rapid changes, relative to a set of inanimate objects, i.e., a set of billiard balls, for instance. Although, one tends habitually to disregard such natural mutations at the level of mathematical (arithmetical) analyses, the rigid, unchangeable structure of natural numbers are more closely respected by the sets of rigid, macroscopic material objects such as a set of billiard balls. Consequently, the traditional concept of natural numbers is lifeless, being devoid of an intrinsic potentiality of an irreversible change. Let us recall here that the theory of fuzzy sets and fuzzy logic is an approach to capture this 'softer' (non-rigid) aspect of the natural systems. Our new results, even in the framework of the standard classical analysis offers an independent new approach to this problem.

In view of the existence of intrinsically dynamic infinitesimals, every real number, and hence every natural number is now identified with a cell like structure (the intrinsically evolving set of infinitesimally close elements, any particular member of which being an approximate evaluation of the given natural number), which experiences a spontaneous fluctuation and irreversible evolution at every finer scale. The scale free number $1_{f}$, for example, now may be more accurately represented, at any given instance (as recorded by an ordinary clock), by the set of a new born living cell, $1_{f}=\{1$ cell $\}$ (of course, $1_{f}$ can represent more complex sets such as a budding flower, or a sapling etc. We are, however, considering here the simplest case.). Now, $1_{f}=1 \pm \eta$, by definition, where $\eta=k t, k$ is an infinitesimal scale factor and $t$ is an $\mathrm{O}(1)$ intrinsic time variable which keeps record of the cell evolution. We note that $\eta$ satisfies eq(4), by definition, so that $k$ must solve eq(3) in $t^{-1}$. Had the fluctuations $\eta$ been reflection symmetric (i.e., $k$ be the reflection symmetric solution (10), the cell like structure would have survived eternally. Because of the time asymmetric solution eq(5), $1_{f}$, however, enjoys a number of evolutionary patterns, depending on the random selection of the \pm sign, as $t \rightarrow 1 / k$. 
Before detailing different evolutionary features, let us first examine more closely how the time asymmetric solution renders $\eta$ dynamic, with a slow, but directed evolution. As indicated, an infinitesimal $\eta$ can always be parametrized as $\eta=k t$, where $t=1 \pm \eta_{1}, \eta_{1}>0$ being another infinitesimal. As seen already, $\eta_{1}$ (and hence $\eta$ ) cannot be static, ordinary constant, and consequently, $t$ must undergo 'infinitely' slow, irreversible evolution towards $1 / k$, because of the cooperative effect of the infintesimally small scale free (random) fluctuations, represented by the time asymmetric solutions (5) and (11). In fact, the solution (11) forces $t$ to change irreversibly towards the forward direction by the applications of infinitesimal scalings, inversions and translations. Let for instance, $t=1-\eta_{1}$. Then infinitesimal fluctuations transform $t$ to $t_{1}=t^{-\alpha}=1+\alpha \eta_{1} \equiv 1+\eta_{2}, \alpha>0$ (by a combination of inversion and scaling). This new value of $t_{1}$ could now continue to grow by analogous applications of scalings of the form $t_{1} \rightarrow t_{2}=t_{1}^{\alpha_{1}}$ and so on, until the successively transformed value of $\eta_{n}$ after $n$th iteration becomes of the order of a small macroscopic variable $\bar{t}(0<\bar{t}<<1)$, which will then increase monotonically (and linearly) till $t=1+\bar{t} \sim 1 / k$. Analogously, $t=1+\eta_{1}$ would also increase towards $1 / k$ by successive applications of infinitesimal scalings and translations. This intrinsic monotonic movement of $t$ must be infinitely slow, since the number of iterations $n$ should be an infinitely large positive integer, for any finite $\alpha$.

Once the intrinsic time like variable $t$ grows to $\mathrm{O}(1 / \mathrm{k})$, so that $\eta=1 \pm \eta^{\prime}$, the cell like structure of of $1_{f}$ would either (a) spontaneously replicate to two daughter cells: $1_{f}(=1+\eta) \rightarrow$ $2_{f}=1_{f}^{\prime}+1_{f}^{\prime}$, where $1_{f}^{\prime}=1+\frac{1}{2} \eta^{\prime}$ is an almost exact replica of $1_{f}$, but for an infinitesimal variation, or (b) annihilate $1_{f}(=1-\eta) \rightarrow 0_{f}$. The second possibility may signify the 'death' of the new born cell, whereas the first case corresponds to the replication of the mother cell into daughter cells by the process of mitotic division. Because of the random occurrence of the \pm sign, the process of celluar division would likely to be halted after a finite number of cell replication when the growing mode (viz., $1_{f}$ with a '+' sign) meets for the first time the '-' sign and is thus transferred to the decaying mode. (The role of the golden mean, and the Fibonacci numbers in cell division would be examined separately. )

To summarize, the scale free natural numbers seems to enjoy living cell like intrinsic dynamical behaviours. One needs, however, to be reminded that the present analysis cannot pretend to be a complete description of the cell division process. The significance of the present discussion is to highlight the importance of the intrinsic dynamics in the contemporary studies of cell division and other biological processes. In fact, one may call a system living whose dynamics is more efficiently controlled by the scale free intrinsic time like component, compared to mere external time treatment, as in vague in the contemporary studies. In this sense, every system is living, in the present extended framework of scale free dynamics. However, one system may be 
more living than other. For example, a billiard ball is material (nonliving) since the intrinsic time and corresponding intrinsic dynamics become relevant only infinitely slowly, making it meaningless in the context of ordinary Newtonian dynamics. However, for a living biological cell, the intrinsic dynamics should become effective even at an order $\mathrm{O}(1)$ of the ordinary time scale.

\section{Newton's first law of motion: An intelligent version}

The first law of motion in classical mechanics states that ' a particle in uniform rectilinear motion or in rest will continue to remain in the state of uniform motion or rest unless disturbed by an externally applied force'. A direct consequence of this law is the following. Let A and B be two points, moving uniformly with speed 1 unit along $x$ axis from the initial points $x=0$ and $x=2$ respectively, in the opposite directions. Then, according to the first law, the two particles would definitely collide at $x=1$.

However, an interesting variation of the first law could be possible in the extended scale free mechanics, incorporating time asymmetric solutions. We note at first that the instantaneous positions of the two particles are given by $x_{A}=t$ and $x_{B}=2-t$ respectively, $t$ being the time variable. Thus as $t \rightarrow 1^{-}, x_{A}=1-\eta$ and $x_{B}=1+\eta, 0<\eta<<1$. Consequently, for an infinitesimal $\eta$, A and B may avoid direct collision, by simply flipping the sign of $\eta$, thereby interchanging the role of $x_{A}$ and $x_{B}$. As a result, $\mathrm{A}$ and $\mathrm{B}$, as they approach towards $x=1$, would jump around each other, and then continue their respective motions uninterrupted, viz., A towards $x=2$ and $\mathrm{B}$ towards $x=0$. One is, therefore, tempted to infer that the

time asymmetric solution instills, so to speak, intelligence into moving particles, leading to an avoidance of the otherwise unavoidable collision. Further, the reason of this avoidance is not due to some external forces, but solely an intrinsic property of the asymmetric solutions of the linear differential equations. The avoidance of an immanent collision, as dictated by the Newton's first law, provides an instance of a 'controlled' behaviour, which along with the intrinsic irreversibility, as pointed out in Sec.2.2, may be considered as the hallmark of an intelligent living system.

\section{Appendix}

An exact (to all orders of $\eta$ ) random solution of eq(3) is obtained when the approximate solution (5) is improved recursively by self-similar correction factors over smaller and smaller scales $\eta, \eta^{2}, \eta^{4}, \ldots$ Let 


$$
\tau_{f}(t)=\left\{\begin{array}{ll}
\tau_{-} & \text {if } t \lesssim 1 \\
\tau_{+} & \text {if } t \gtrsim 1
\end{array}, \tau_{-}\left(t_{-}\right)=\left(1 / t_{+}\right) f_{-}(\eta), \tau_{+}\left(t_{+}\right)=t_{+}\right.
$$

We consider the nontrivial part only. One obtains

$$
t_{-} \frac{\mathrm{d} \tau_{-}}{\mathrm{d} t_{-}}=\tau_{-}\left(\frac{t_{-}}{t_{+}}-t_{-} \frac{f_{-}^{\prime}}{f_{-}}\right)
$$

where $f^{\prime}=\frac{\mathrm{d} f}{\mathrm{~d} \eta}$. As a result, $\tau_{-}$solves eq(13) exactly provided $f_{-}$solves exactly the self-similar equation

$$
t_{-}^{\prime} \frac{\mathrm{d} f_{-}}{\mathrm{d} t_{-}^{\prime}}=f_{-}
$$

in the smaller scale variable $\ln \left(1 / t_{-}^{\prime}\right)=\ln \left(1-\eta^{2}\right)^{-1}$. The exact solution is thus obtained as an infinite product

$$
\tau_{=} \frac{1}{t_{+}} \frac{1}{t_{+}^{\prime}} \frac{1}{t_{+}^{\prime \prime}} \ldots
$$

where $t_{+}^{\prime}=\left(1+\eta^{2}\right), t_{+}^{\prime \prime}=\left(1+\eta^{4}\right)$ etc. One therefore reproduces the standard solution $\tau_{-}=1-\eta$ (since $t_{+} t_{+}^{\prime} \ldots=(1-\eta)^{-1}$ ), when $\eta$ is an ordinary real variable with exact values. In the present extended treatment $\eta$, however, is a small random variable. The solution $\tau_{-}$could therefore be interpreted as an infinite multiplicative process, the self-similar factors of which may be treated as independent random variables defined recursively via eq(3) and eq(A4). Maintaining continuity of the second derivative of $\tau_{f}$ at $\eta=0$, in the ordinary real calculus sense, would be impossible.

\section{References}

[1] A Robinson, Nonstandard analysis, North-Holland, Amsterdam, (1966).

[2] L Nottale, Fractal Space Time and Microphysics, World Scientific, Singapore (1993).

[3] D P Datta, A new class of scale free solutions to linear ordinary differential equations and the universality of the Golden Mean $\frac{\sqrt{5}-1}{2}=0.618033 \ldots$. Chaos, Solitons \& Fractals,2003, 17, 621-630.

[4] D. P. Datta, The Golden mean, scale free extension of Real number system, fuzzy sets and $1 / f$ spectrum in Physics and Biology, Chaos, Solitons $\&$ Fractals, 2003, 17, 781-788.

[5] R. Penrose, Shadows of the Mind, Oxford, Oxford University Press, 1994. 
[6] G. N. Ord, Fractal spacetime: a geometric analog of relativistic quantum mechanics, $J$. Phys. A Math. Gen., 1983, 16, 1869-1884.

[7] M S El Naschie, Modular groups in Cantorian $\mathcal{E}^{\infty}$ high energy physics, Chaos, Solitons $\mathscr{E}$ Fractals, 2003, 16, 353-366.

[8] M S El Naschie, O E Rössler, I Prigogine, Quantum Mechanics, diffusion and chaotic fractals, Oxford, Pergamon, 1995.

[9] B G Sidharth, Space time as a random heap, Chaos, Solitons \& Fractals, 2001, 12, 173-178.

[10] B J West and W Deering, Fractal physiology for physicists:Levi statistics, Physics Report, 1994, 246,1-100.

[11] E Goldfain, Renormalization group and the emergence of random fractal topology in quantum field theory, Chaos, Solitons $\&$ Fractals, 2003, to appear.

[12] I Prigogine, The arrow of time, available at http://www.icra.it/Publications/Books/Prigogine/ Prigogine_motivation.htm

[13] M S El Naschie, Quantum gravity from descriptive set theory, Chaos, Solitons $\&$ Fractals, in press, 2003.

[14] M S El Naschie, A review of E-infinity theory and the mass spectrum of high energy particle physics, Chaos, Solitons \& Fractals, in press, 2003. 